\title{
Electrocardiographic Analysis of Repolarization Changes in South Indian Children with Kawasaki Disease after the Acute Phase of Illness
}

\author{
Siddhartha Reddy, ${ }^{1}$ Maneesh Rai, ${ }^{2}$ Ravi Raj Singh Chouhan, ${ }^{3}$ \\ Suchetha Rao ${ }^{(D)}{ }^{4}$ and Nutan Kamath ${ }^{4}{ }^{4}$ \\ ${ }^{1}$ Fernandez Hospital, Hyderabad, Telangana 500034, India \\ ${ }^{2}$ Department of Cardiology, Kasturba Medical College, Manipal Academy of Higher Education, Mangalore, Karnataka 575001, India \\ ${ }^{3}$ Kasturba Medical College, Manipal Academy of Higher Education, Mangalore, Karnataka 575001, India \\ ${ }^{4}$ Department of Paediatrics, Kasturba Medical College, Manipal Academy of Higher Education, Mangalore, Karnataka 575001, India
}

Correspondence should be addressed to Nutan Kamath; nutan.kamath@manipal.edu

Received 19 February 2018; Revised 16 May 2018; Accepted 3 June 2018; Published 2 July 2018

Academic Editor: Samuel Menahem

Copyright ( 2018 Siddhartha Reddy et al. This is an open access article distributed under the Creative Commons Attribution License, which permits unrestricted use, distribution, and reproduction in any medium, provided the original work is properly cited.

\begin{abstract}
Background. Cardiac involvement in children with Kawasaki disease (KD) may present with repolarization abnormalities which are associated with increased risk of ventricular arrhythmias and sudden cardiac events. Methods. Twenty children with history of $\mathrm{KD}$ without cardiac involvement in the acute phase were recruited along with age and sex-matched controls. Twelve-lead ECG was obtained from both groups using CARDIART 610T ECG system at $25 \mathrm{~mm} / \mathrm{sec}$ and $50 \mathrm{~mm} / \mathrm{sec}$ paper speed. ECG was repeated in 19 children in the study group after $9 \pm 2$ months. Measurements (QT dispersion (QTd), T-wave peak to end (Tp-Te) interval, and $\mathrm{Tp}-\mathrm{Te} / \mathrm{QT}$ ratio) were made using standard digital calipers. Statistical analysis was performed with student $t$-test and analysis of variance (ANOVA) using SSPS version 17.0. Results. The mean value of QTd in the first ECG in cases was significantly high: $43.15 \pm 14.13$ versus $29.47 \pm 8.637$ in the controls $(p=0.001)$. The follow-up ECG in 19 cases showed a mean value of $46.26 \pm 16.25$ versus $43.89+-14.53$ at baseline $(p=0.440)$. QTd was increased in the follow-up ECG but was not statistically significant. There was no statistical significance seen in the Tp-Te interval and Tp-Te/Qt ratio as observed in Lead II and Lead V5. Conclusion. Significant increase in the QTd in children with KD indicates repolarization changes in the myocardium even in the absence of clinical carditis. The persistence of this change on follow-up could indicate a possible increased risk for ventricular arrhythmia and warrants long term assessment of the cardiovascular status.
\end{abstract}

\section{Introduction}

Kawasaki disease (KD) is an acute, self-limiting, febrile illness that occurs predominantly in children with pathology demonstrating vasculitis of small and medium size blood vessels with a predilection for coronary arteries [1]. It is complicated by either clinical or subclinical myocarditis in the acute stage and can lead to histological changes of the myocardium such as interstitial fibrosis. These abnormalities may persist even after the acute phase without the involvement of the coronary arteries [2].
Myocardial damage results in altered electrical potential distributions and repolarization changes manifesting as prolonged Qt dispersion and electrophysiological changes in Twaves $[3,4]$.

$\mathrm{T}$ peak is a marker of epicardial repolarization while $\mathrm{T}$ end is believed to represent completion of repolarization of the mid-myocardial cells. The interval between $\mathrm{T}$ peak and $\mathrm{T}$ end provides a measure of transmural dispersion of repolarization which can be used as a tool for detection of life threatening arrhythmias [5].

Only a few studies have demonstrated these repolarization changes after the acute phase of illness. The long 
term impact on myocardial electrical stability after $\mathrm{KD}$ is not well understood especially in children with no cardiac involvement during the acute phase. So a prospective cohort study was conducted on 20 children with history of KD for the analysis of repolarization changes on ECG.

\section{Methods}

A hospital-based prospective study was conducted at a tertiary care centre between October 2014 and September 2016. Children with history of KD diagnosed at least 3 months prior were included in the study. Patients who fulfilled the inclusion criteria were recruited into the study after getting clearance from the institutional ethics committee. Informed consent was obtained from either of the parents of the child and a semistructured proforma was prepared to record all data.

A 12-lead ECG was obtained from each subject after a period of 5 minutes of rest using CARDIART 6108T ECG system at $25 \mathrm{~mm} / \mathrm{sec}$ and $50 \mathrm{~mm} / \mathrm{sec}$ paper speed. Measurements were made manually using STANDARD DIGITAL CALIPERS (AEROSPACE, China). The following variables were measured using the calipers

(1) Qt dispersion (QTd): defined as the difference between maximum and minimum QT interval of a 12-lead ECG.

(2) T peak to $\mathrm{T}$ end (Tpe) interval: the peak of the Twave as defined as a point of highest amplitude of the $\mathrm{T}$-wave deflection and the end as a point where the tangent on descending limb of T-wave intersects the isoelectric line.

(3) $\mathrm{T}$ peak to $\mathrm{T}$ end/QT(Tpe/QT) ratio.

ECGs with low amplitude or unreliable $\mathrm{T}$-waves and $\mathrm{U}$ waves were excluded from analysis. The mean QTd were calculated from all the 12 leads in the study group and compared with the control group. Tpe and Tpe/QT ratio were measured in one of the limb leads (II) and chest leads (V5) and the mean calculated values were compared between the groups.

Blood pressure was recorded for all the children included in the study using a standard mercury sphygmomanometer with appropriate cuff size. A repeat ECG was obtained from the study group after a period of $9 \pm 2$ months and the same variables were measured.

ECG was interpreted with the guidance of a cardiologist with fellowship in cardiac electrophysiology and a 2D ECHO was performed on all children with history of KD by the same cardiologist to look for coronary artery abnormalities. Out of the 696 leads obtained, 71 leads had to be excluded from analysis because of the poor $\mathrm{T}$-wave formation and presence of $\mathrm{U}$ waves.

Collected data were analyzed using statistical package for social sciences (SPSS) version 17.0. The data was presented as mean and standard deviations. Statistical analysis for difference in mean value was performed using student $t$-test. Correlations were analyzed by analysis of variance (ANOVA). $P$ value of less than 0.05 was considered significant.

\section{Results}

In children with history of Kawasaki disease, QT dispersion is significantly increased compared to the control group. The mean value of QTd in the first ECG in children with $\mathrm{KD}$ was $43.89 \pm 14.529 \mathrm{msec}$ when compared to $29.47 \pm 8.637 \mathrm{msec}$ in the control group.

The follow-up ECG also showed similar result with a mean value of $46.26 \pm 16.254 \mathrm{msec}$ in the study group when compared to the control group (Figure 1(a)). QT dispersions were increased in the follow-up ECG when compared to the first ECG in the control group but are not statistically significant (Figure 1(b)).

There was no statistical significance observed in the TpTe interval in cases when compared to controls, Lead II $(68.08 \pm 17.11$ versus $64.97 \pm 14.185 ; p=0.099)$, and Lead V5 (70.21 \pm 15.293 versus $63.37 \pm 10.877 ; p=0.509)$ (Figures $1(\mathrm{c})$ and $1(\mathrm{~d}))$. The same was observed with $\mathrm{Tp}-\mathrm{Te} / \mathrm{QT}$ ratio in cases compared to controls (Lead II- $0.21 \pm 0.05$ versus $0.21 \pm$ 0.034; $p=0.138$; Lead V5- $0.22 \pm 0.05$ versus $0.20 \pm 0.031$; $p=0.734$ (Figures $1(\mathrm{e})$ and $1(\mathrm{f})$ ). The follow-up ECG in cases also showed no statistical difference in the Tp-Te interval and $\mathrm{Te} / \mathrm{QT}$ ratio.

\section{Discussion}

Kawasaki disease $(\mathrm{KD})$ is an acute, self-limiting, febrile illness that occurs predominantly in children with pathology demonstrating vasculitis of small and medium size blood vessels with a predilection for coronary arteries. It is complicated by either clinical or subclinical myocarditis in the acute stage and can lead to histological changes of the myocardium such as interstitial fibrosis.

We attempted to study the hypothesis that, in children with $\mathrm{KD}$ without overt coronary artery involvement, myocardial abnormalities may persist even after acute phase which is reflected on surface ECG as repolarization changes. The study was conducted on 20 children with history of typical $\mathrm{KD}$ without coronary artery involvement in the acute phase and treated with $2 \mathrm{~g} / \mathrm{kg}$ of IVIG within 10 days of onset of illness (diagnosed based on American Heart Association (AHA) criteria) and an equal number of age and sex-matched controls.

QT interval dispersion (QTd) represents a general abnormality in repolarization. Increased QT dispersion has been demonstrated in various cardiac diseases. Data obtained from almost 7000 patients with cardiac disorders like myocardial infarction, cardiomyopathies, and long QT syndrome have shown that there is definite evidence of increased QT dispersion in these cardiac disorders [6].

Our study showed significant difference in the QT dispersion in children with KD when compared to the control group. The follow-up ECG which was done $9 \pm 2$ months showed increased QT interval dispersion compared to the initial ECG, but the increase was not statistically significant.

In the year 1999, a study was conducted by Osada et al. [7] wherein they have studied the QTd. Children with KD were grouped based on the degree of involvement of coronaries during the acute phase. It was found that QT 


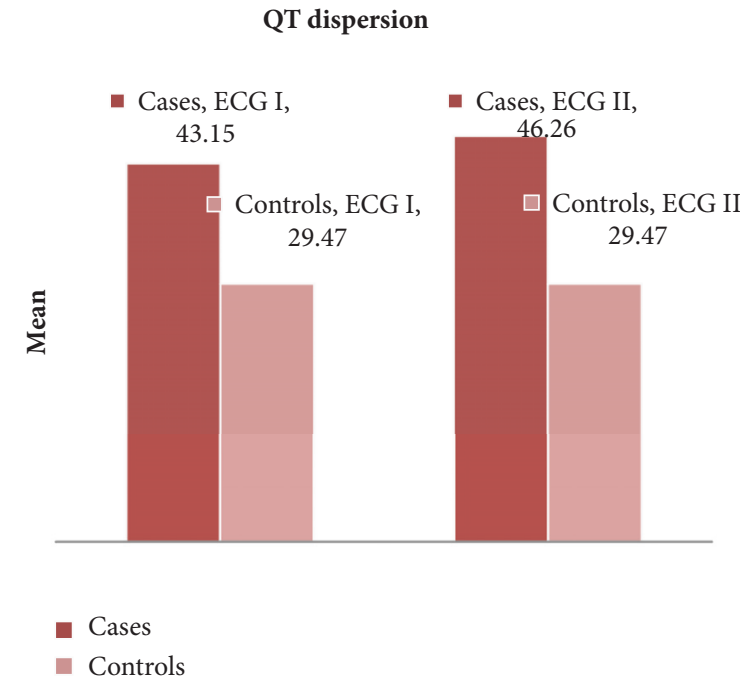

(a) Comparison of mean QTd in children with KD with controls

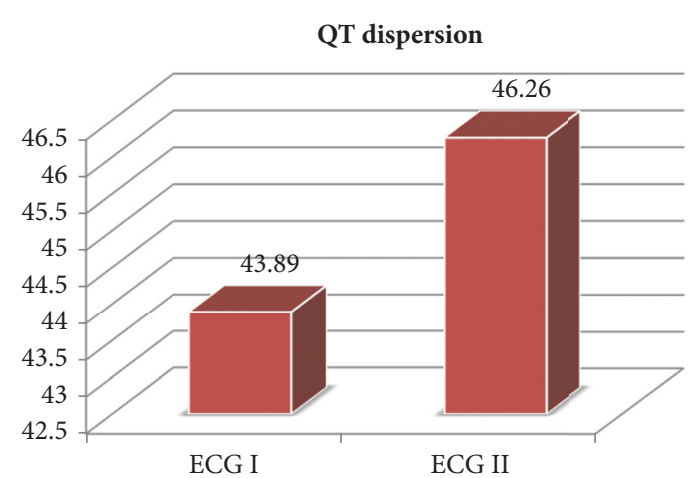

- QT dispersion

(b) Comparison of mean in children with $\mathrm{KD}$ with follow-up ECG

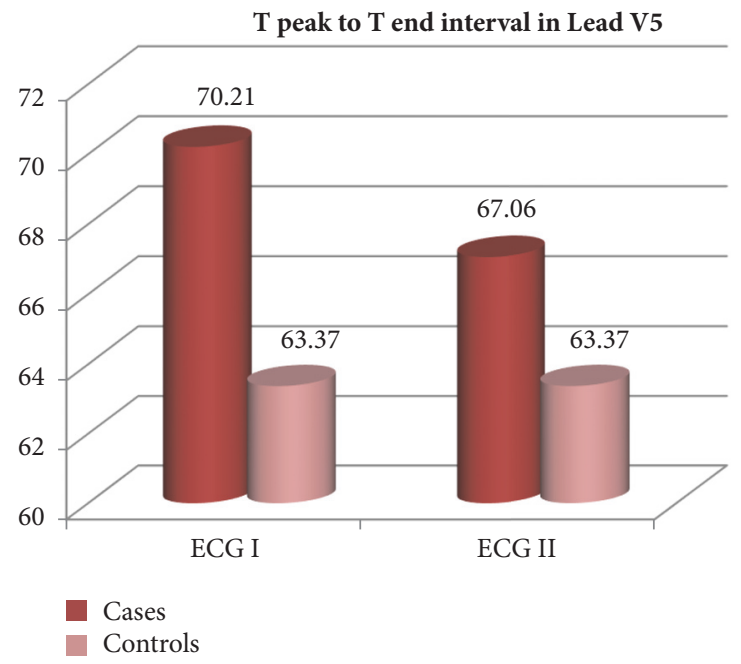

(d) Comparison of mean $\mathrm{T}$ peak to $\mathrm{T}$ end interval (Lead V5)

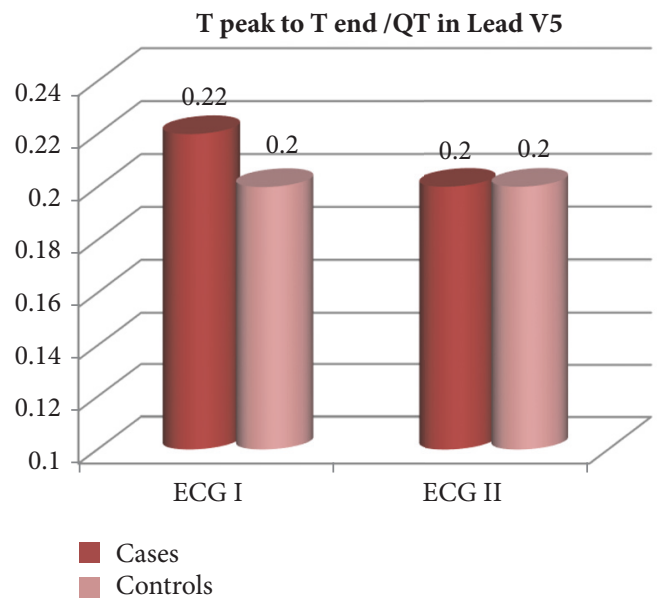

(f) Comparison of mean $\mathrm{T}$ peak to $\mathrm{T}$ end/QT (Lead V5)

Figure 1 
interval dispersions were proportionately increased with the degree of involvement of coronaries. The main drawback of the study was lack of control group to compare the results.

Three years later Dahdah et al. [3] conducted a similar study. Children with history of KD were categorized into three different groups not just based on the degree of involvement of coronaries but depending on the degree of resolution of coronary aneurysms. The study concluded that QTd and QTcd (corrected Qt interval dispersion) were increased in patients who had persistent coronary artery aneurysm.

In 2008, Ghelani et al. [8] performed a study on Indian population which included children with history of KD with no overt coronary artery abnormalities. QTd was measured and compared with the control group. The study showed significant increase in QTd when compared to the control group thereby concluding that there is evidence of heterogeneous ventricular repolarization in children with $\mathrm{KD}$ who had no apparent coronary involvement during the acute phase.

Similarly, in 2014, Gupta et al. conducted a study on a cohort including 30 children with history of $\mathrm{KD}$ and measured QTd. Though it was not the main objective of the study they have concluded that study population showed increased QTd when compared to the control group.

Recently a study conducted by Parihar et al. [9] on a cohort of 20 children diagnosed to have KD with transient coronary artery abnormalities during the acute phase concluded that there is no significant increase in QTd which is contradictory to the above-mentioned studies.

4.1. T Peak to T End and T Peak to T End/QT. Apart from QT dispersion, we have also studied the ventricular repolarization changes, namely, $\mathrm{T}$ peak to $\mathrm{T}$ end interval and $\mathrm{T}$ peak to $\mathrm{T}$ end/QT ratio. These variables have been used extensively in predicting the arrhythmic risk in various cardiac disorders like myocardial infarction, hypertrophic cardiomyopathy, and pulmonary embolism (PE) [10]. It has been proposed that Tpe and Tpe/QT variables represent a more precise tool for measuring the ventricular repolarization as these variables are less dependent on heart rate, autonomic changes, and QRS duration [11].

Evidence supporting these parameters as a prognostic tool in forecasting the risk of arrhythmias have been provided under congenital and acquired long Qt, hypertrophic cardiomyopathy, and Brugada syndrome [12]. A systematic review and meta-analysis also concluded that $\mathrm{T}$ peak to $\mathrm{T}$ end interval is a useful risk stratification tool in various cardiac illnesses [13].

In a study done by Abdullah Icli et al. [14] it was proposed that Tpe interval can be used as a prognostic tool as risk stratification in patients with acute PE. A study conducted by Hamid Amoozar et al. [15] on 30 children with $\mathrm{KD}$, concluded that $\mathrm{Qt}$ interval and $\mathrm{T}$ peak to $\mathrm{T}$ end interval were significantly increased during the acute phase of illness. In another study conducted by Masayuki Fujino, it was concluded that Tpe/QT ratio is useful for evaluating severity of vasculitis and myocarditis associated with $\mathrm{KD}$ [16].

The ratio between the Tpe interval and QT interval (Tpe/QT) has been proposed as a noninvasive marker of arrhythmic risk along with Tpe interval, while QT interval and Tpe interval vary with various body sizes Tpe/QT remains relatively constant. It was also reported to be stable with varied heart rates [17]. Our study showed no significant changes in these variables in the study group when compared to the control group.

\section{Conclusion}

There is evidence of repolarization changes in the myocardium in children with history of $\mathrm{KD}$ without cardiac involvement in acute stage even after treatment with IVIG/Aspirin in recommended doses. This cohort may be at risk of developing long term complications like arrhythmias. This cohort requires long term assessment and follow-up to identify the risk of myocardial dysfunction. Further studies are warranted on a larger sample size to identify the significance of these repolarization parameters in forecasting the arrhythmic risk.

\section{Data Availability}

The data used to support the findings of this study are available from the corresponding author upon request.

\section{Disclosure}

An earlier version of this work was presented at Indian Rheumatology Association Conference "IRACON” 2016.

\section{Conflicts of Interest}

The authors declare that there are no conflicts of interest regarding the publication of this paper.

\section{References}

[1] T. Kawasaki and S. Naoe, "History of Kawasaki disease," Clinical and Experimental Nephrology, vol. 18, no. 2, pp. 301-304, 2014.

[2] M. Takeuchi, T. Matsushita, S. Kurotobi, T. Sano, S. Kogaki, and K. Ozono, "Application of signal-averaged electrocardiogram to myocardial damage in the late stage of Kawasaki disease," Circulation Journal, vol. 70, no. 11, pp. 1443-1445, 2006.

[3] N. S. Dahdah, E. Jaeggi, and A. Fournier, "Electrocardiographic depolarization and repolarization: Long-term after Kawasaki disease," Pediatric Cardiology, vol. 23, no. 5, pp. 513-517, 2002.

[4] P. D. Higham and R. W. Campbell, "QT dispersion.," Heart, vol. 71, no. 6, pp. 508-510, 1994.

[5] T. N. Bachmann, M. W. Skov, P. V. Rasmussen et al., "Electrocardiographic Tpeak-Tend interval and risk of cardiovascular morbidity and mortality: Results from the Copenhagen ECG study," Heart Rhythm, vol. 13, no. 4, pp. 915-924, 2016.

[6] M. Malik and V. N. Batchvarov, "Measurement, interpretation and clinical potential of QT dispersion," Journal of the American College of Cardiology, vol. 36, no. 6, pp. 1749-1766, 2000.

[7] M. Osada, Y. Tanaka, T. Komai et al., "Coronary arterial involvement and QT dispersion in Kawasaki disease," American Journal of Cardiology, vol. 84, no. 4, pp. 466-468, 1999.

[8] S. J. Ghelani, S. Singh, and R. Manojkumar, "QT interval dispersion in North Indian children with Kawasaki disease without overt coronary artery abnormalities," Rheumatology International, vol. 31, no. 3, pp. 301-305, 2011. 
[9] M. Parihar, S. Singh, P. Vignesh, A. Gupta, and M. Rohit, "Mid-term Risk for Subclinical Atherosclerosis and Chronic Myocarditis in Children with Kawasaki Disease and Transient Coronary Abnormalities," Pediatric Cardiology, vol. 38, no. 6, pp. 1123-1132, 2017.

[10] J. Castro Hevia, C. Antzelevitch, F. Tornés Bárzaga et al., "Tpeak-Tend and Tpeak-Tend Dispersion as Risk Factors for Ventricular Tachycardia/Ventricular Fibrillation in Patients With the Brugada Syndrome," Journal of the American College of Cardiology, vol. 47, no. 9, pp. 1828-1834, 2006.

[11] R. Panikkath, K. Reinier, A. Uy-Evanado et al., "Prolonged tpeak-to-tend interval on the resting ECG is associated with increased risk of sudden cardiac death," Circulation: Arrhythmia and Electrophysiology, vol. 4, no. 4, pp. 441-447, 2011.

[12] C. Antzelevitch, G.-X. Yan, and W. Shimizu, "Transmural dispersion of repolarization and arrhythmogenicity: The Brugada syndrome versus the long QT syndrome," Journal of Electrocardiology, vol. 32, pp. 158-165, 1999.

[13] G. Tse, M. Gong, W. T. Wong et al., "The T peak - T end interval as an electrocardiographic risk marker of arrhythmic and mortality outcomes: A systematic review and meta-analysis," Heart Rhythm, vol. 14, no. 8, pp. 1131-1137, 2017.

[14] A. Icli, M. Kayrak, H. Akilli et al., "Prognostic value of TpeakTend interval in patients with acute pulmonary embolism," BMC Cardiovascular Disorders, vol. 15, no. 1, article no. 99, 2015.

[15] H. Amoozgar, M. Ahmadipour, and A. Amirhakimi, "QT dispersion and T wave peak-to-end interval dispersion in children with Kawasaki disease," International Cardiovascular Research Journal, vol. 7, no. 3, pp. 99-103, 2013.

[16] M. Fujino, T. Hata, M. Kuriki et al., "Inflammation aggravates heterogeneity of ventricular repolarization in children with kawasaki disease," Pediatric Cardiology, pp. 1268-1272, 2014.

[17] P. Gupta, C. Patel, and H. Narayanaswamy, "T(p-e)/QT ratio as an index of arrhythmogenesis," Journal of Electrocardiology, vol. 41, no. 6, pp. 567-574, 2008. 


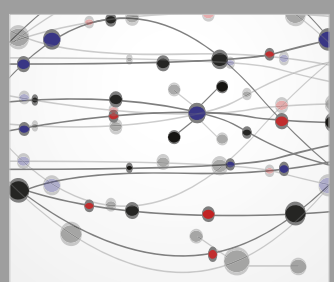

The Scientific World Journal
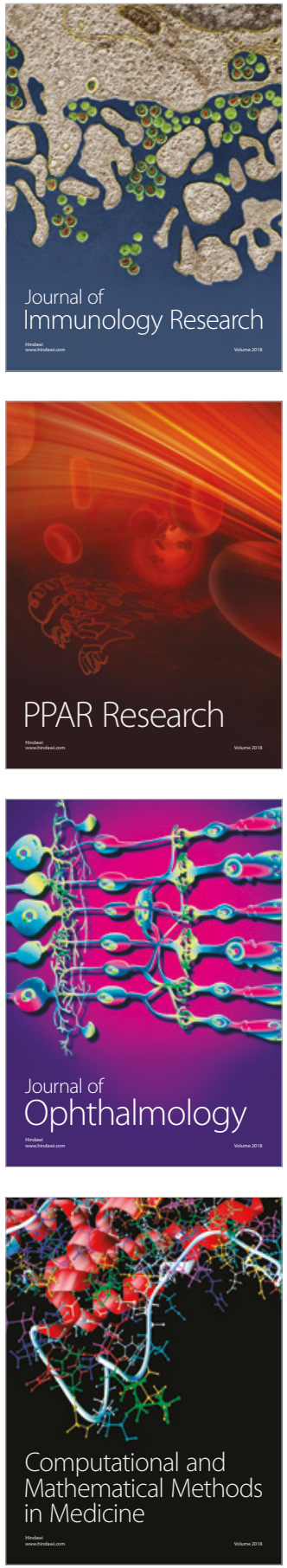

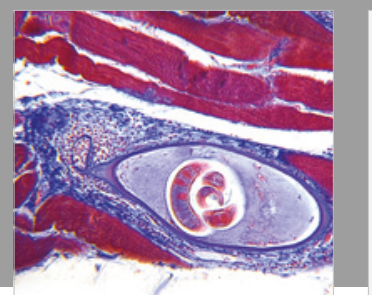

Gastroenterology Research and Practice

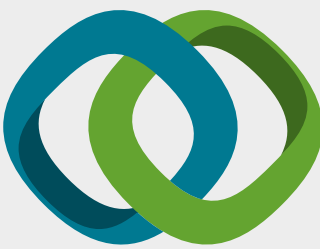

\section{Hindawi}

Submit your manuscripts at

www.hindawi.com
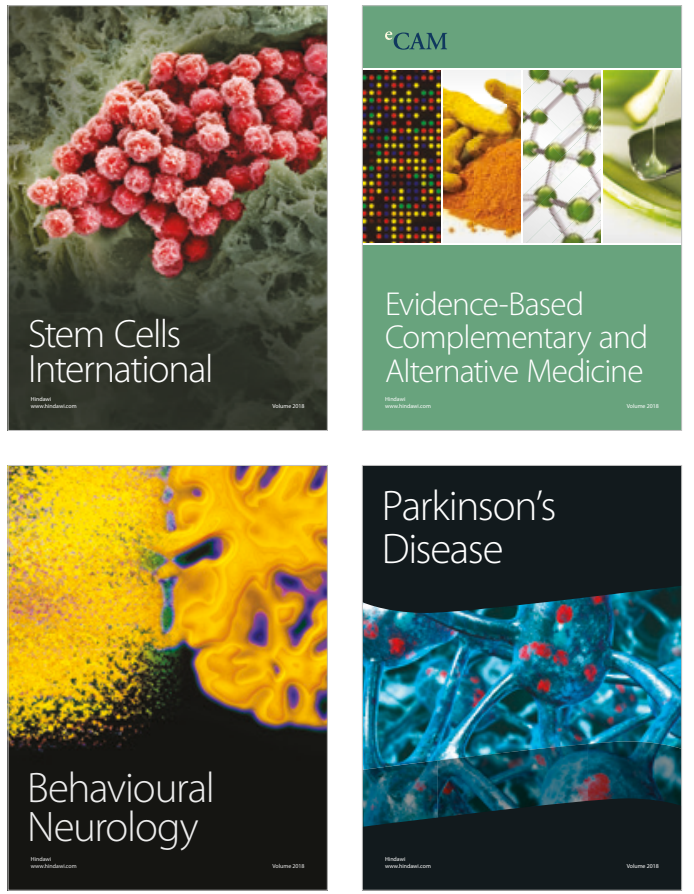

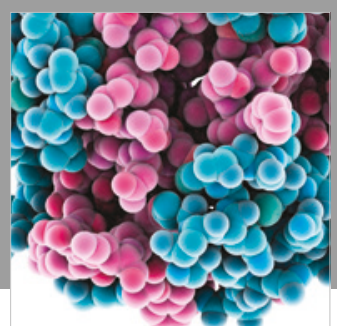

ournal of

Diabetes Research

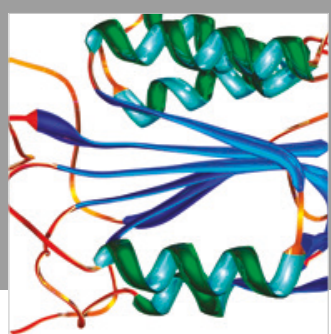

Disease Markers
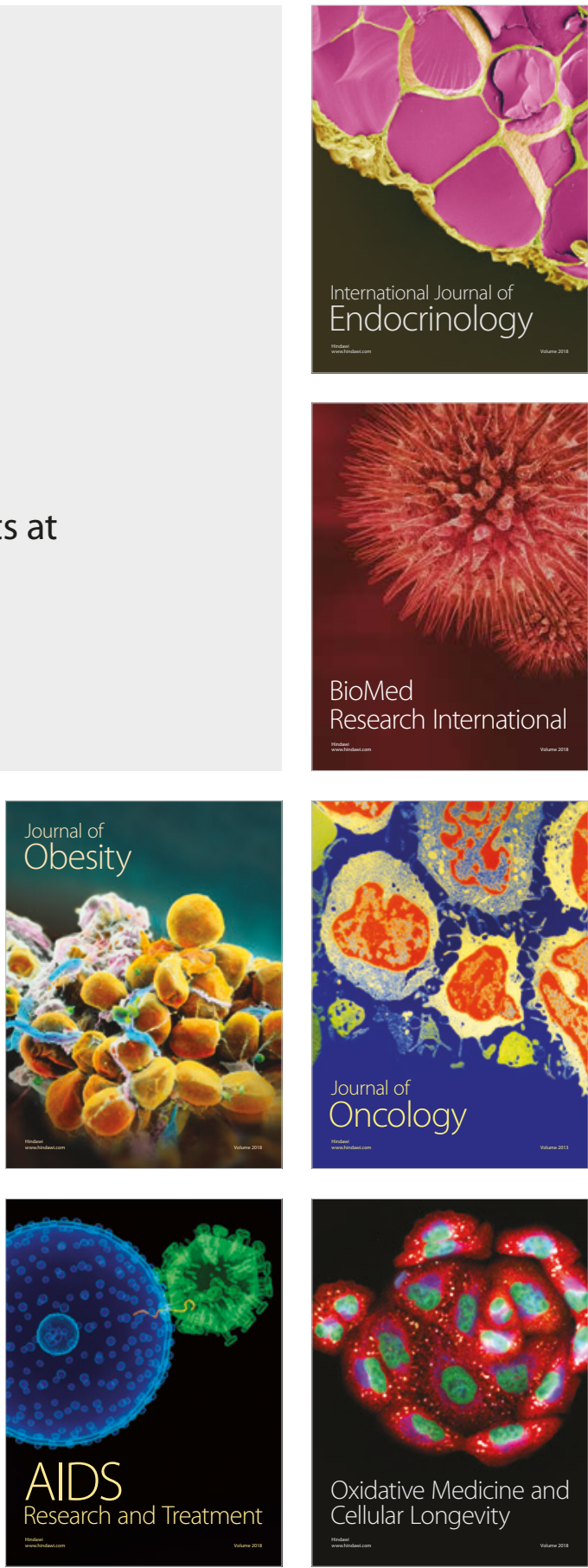